\title{
Frontières
}

\section{La mort du cygne}

À propos d'un madrigal italien du XVI ${ }^{\mathrm{e}}$ siècle

\section{Frédérique Riedlin et Patrick Ténoudji}

Volume 20, numéro 2, printemps 2008

Les musiques et la mort

URI : https://id.erudit.org/iderudit/018332ar

DOI : https://doi.org/10.7202/018332ar

Aller au sommaire du numéro

\section{Éditeur(s)}

Université du Québec à Montréal

ISSN

1180-3479 (imprimé)

1916-0976 (numérique)

Découvrir la revue

Citer cet article

Riedlin, F. \& Ténoudji, P. (2008). La mort du cygne : à propos d'un madrigal italien du XVI ${ }^{\mathrm{e}}$ siècle. Frontières, 20(2), 37-43. https://doi.org/10.7202/018332ar
Résumé de l'article

Cet article, qui analyse un madrigal polyphonique italien de 1539, contextualise le thème de la mort dans la chanson italienne $\mathrm{du} \mathrm{xvi}^{\mathrm{e}}$ siècle dans la vie philosophique, sociale, politique et religieuse des cours de Ferrare et Florence entre 1520 et 1550. En s'appuyant sur les concepts psychanalytiques de " jouissance " et de " chose ", il aborde la manière singulière dont le madrigal traite de la mort à travers un anéantissement voluptueux et rémissible du sujet, et dans un rapport initiatique privilégié à l'au-delà.
Ce document est protégé par la loi sur le droit d'auteur. L'utilisation des services d’Érudit (y compris la reproduction) est assujettie à sa politique d'utilisation que vous pouvez consulter en ligne.

https://apropos.erudit.org/fr/usagers/politique-dutilisation/ 


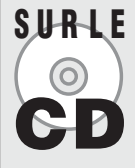

acques Arcadelt et los (1539) Crump, Paul Hillier)

Durée: $1 \min 59$ s

Cet article, qui analyse un madrigal polylienne du XVIe siècle dans la vie philosode la manière singulière psychanalyse - Arcadelt-D'Avalos - chose Abstract

This paper abou political and religious life of the courts of Ferrara and "joy" and subject, (

Keywords: madrigal - Italy psychoanalysis - Arcadelt - D'Avalos acan - academies - aristocracy thing - joy - death.

\section{La mort du cygne À propos d'un madrigal italien du XVle siècle}

\section{Frédérique Riedlin, comédienne, apprentie psychanalyste, Fédération européenne de psychanalyse, Strasbourg. \\ Patrick Ténoudji, \\ maître de conférences, Université Marc-Bloch, CNRS UMR 7043, Sociétés et cultures en Europe, Strasbourg.}

Le madrigal dont nous allons parler appartient à la première génération italienne du genre italien. Cette musique polyphonique, à la fois égalitaire et aristocratique, consistait, entre 1520 et 1570 , à chanter à quatre voix égales un poème amoureux. Les voix sont égales à plusieurs titres: deux hommes, deux femmes, chaque voix est responsable du texte entier. Dans le Concert champêtre, anonyme italien $\mathrm{du}$ Musée de Bourges, quatre pratiquants assis en cercle ${ }^{1}$, coiffés, tête baissée, laissent supposer une pratique sans spectateurs, sotto voce $e^{2}$, a capella, dans un lieu solitaire ou privé. Dans Le concert du Titien (1575 Florence, Palais Pitti), les changements de mesure sont discrètement touchés (c'est le tactus) par chaque interprète, de deux doigts de la main droite sur l'épaule gauche de leur voisin ou voisine, une privauté sans autre exemple alors. Les hommes représentés dans la camera di musica d'un palais princier restent couverts devant le prince : or seul un Grand d'Espagne ou un Pair de France avait ce droit.
À Ferrare ou Florence, plusieurs indices, dont des images ${ }^{3}$, nouvelles ${ }^{4}$ et traités de savoir vivre ${ }^{5}$, sur la noblesse ${ }^{6}$ ou la musique $^{7}$ lient ces madrigaux au phénomène des académies ${ }^{8}$ des palais princiers de ces cités italiennes. Ces sources laissent supposer que la pratique fut même au départ l'exclusivité de ces cénacles, composés exclusivement d'aristocrates (les princes régnants et leur famille) et de lettrés (peintres, poètes, musiciens, philosophes), qu'on appelait «académies» à l'imitation des écoles de philosophie grecques. Réunissant par cooptation les mieux nés et les mieux disants, pensants ou chantant, ces institutions étaient les lieux d'éducation des enfants des princes.

Cela a une dimension politique et même, grâce à la musique, une dimension sacrée. D'abord, cette exclusivité réaffirme l'excellence de l'aristocratie. En politique, cette alliance des meilleurs et des mieux nés représenta un temps l'espoir d'une alternative à l'absolutisme en politique: dans cette société hiérarchisée, il est frappant de constater l'équivalence entre hommes et femmes et entre princes et serviteurs - les artistes: les académies élevaient les princes à la hauteur des poètes, et réciproquement. Cette égalité entre "égaux meilleurs", reprise de Platon ${ }^{9}$, permettait au groupe de dépasser le niveau de la commune 
humanité, et d'approcher celui des choses divines: ces gens étaient au-delà des conventions. Les académies offraient aussi une alternative au monopole de l'éducation scolastique (un "lecteur», des auditeurs) dans les universités (et à celui des Jésuites sur l'éducation des princes). Enfin, dans la rhétorique de la Renaissance, cette formation circulaire représente en raccourci la société tout entière (la présence féminine est donc indispensable) et la sphère le cosmos: la dimension sonore permet à deux éléments spatialement opposés mais complémentaires (ici, le masculin et le féminin) de former une sphère, une figure $\mathrm{du}$ monde, de faire harmonie (armonia, concordia discors, Horace).

Comme l'a observé Newcomb (1980), à Florence et Naples, le madrigal est appelé musica reservata et, à Ferrare, musica secreta: il est réservé à ses pratiquants, à ceux qui savent l'entendre (voir le prologue de L'Amfiparnaso de Vecchi, 1596). Dans les traités musicaux de la fin du Moyen Âge, «la musique qu'on entend » (Chailley, 1950) et, dans les cours du début $\mathrm{du} \mathrm{XVI} \mathrm{X}^{\mathrm{e}}$ siècle, toute musique instrumentale ou chant monodique étaient considérées comme du "bruit». La musique était d'abord quelque chose d'inaudible. Le caractère circulaire et fermé aux spectateurs de la première génération du madrigal est à cet égard un trait essentiel : ainsi, les innovations instrumentales et représentatives apportées à la fin du siècle au madrigal par Monteverdi ont été dénoncées par les vieux nostalgiques des académies, tel l'academico Artusi en 1596, comme l'intromission du bruit dans la musique: après un demi-siècle, l'enjeu était encore important!

La primauté de ce modèle aristocratique dura deux générations. Le christianisme militant du concile de Trente (1545-1563) et la montée de l'absolutisme, qui correspond à une alliance des monarques et du peuple contre l'aristocratie (voir le Voyage en Angleterre de Tocqueville, 1833), recouvrirent cette alliance des mieux nés et des mieux disants ${ }^{10}$.

Il manque à ce tableau la dimension essentielle de la jouissance, que la psychanalyse va nous aider à exprimer. Le thème de la mort est très souvent présent dans ces madrigaux, mais elle y est traitée d'un façon qui interpelle la psychanalyse sur un de ses terrains familiers. Les outils de l'analyse musicale et littéraire et ceux de la psychanalyse nous permettent d'aborder le madrigal dans la complexité de ce dont il joue, la présence comme absence, le silence comme son, l'égalité et la hiérarchie, la mort comme petite mort, jusque dans sa position d'intermédiaire du groupe de musiciens comme «corps» collectif dominant, où règne l'égalité des meilleurs. L'essentiel restera insaisissable, comme la Chose pour le parlêtre en psychanalyse lacanienne, cette plénitude promise au cœur de la jouissance, qui s'avère être un point aveugle du sens, à la fois point mort et point de perspective. Quel est donc ce corps à plusieurs voix équivalentes qui semble s'extasier de mourir et vouloir tisser pour les autres, et contre le bruit des autres le contrepoint qui rendrait audible la secrète architecture d'un monde créé par Dieu?

\section{LA MORT DANS IL BIANCO E DOLCE CIGNO D'ARCADELT ET AVALOS: AU SOMMET DU MALAISE ET DU PLAISIR}

Le madrigal a pour titre Il Bianco e dolce cigno (Le blanc et doux cygne). Son auteur, le comte D'Avalos, est un modèle d'académicien : un prince, réputé le meilleur poète de son temps. Le compositeur est Jacques Arcadelt (c. 1507-1568), l'année de publication 1539. Le sujet était un classique du genre: un cygne blanc meurt chantant, mais désespéré, un poète meurt pleurant, mais content. Oiseau consacré à Apollon, le cygne en a reçu le don de pressentir l'avenir: d'où son désespoir; mais pour D'Avalos, la différence entre l'oiseau et le poète est liée à la croyance, nourrie de christologie et de platonisme, que pour les meilleurs la mort n'est pas une fin. La pratique du madrigal a pour fonction de « représenter», au sens fort du mot à la Renaissance, cette idée: la présenter réellement à nouveau. Le poète se compare au Socrate de Phédon présentant sa mort prochaine à ses disciples :

À ce que je vois vous me croyez

inférieur aux cygnes pour la

divination; quand ils sentent

approcher l'heure de la mort, les

cygnes chantent plus mélodieusement qu'ils ne l'ont jamais fait, parce qu'ils sont joyeux de s'en aller chez le dieu dont ils sont les serviteurs. [...] Mais les hommes, par suite de la crainte de la mort, vont jusqu'à calomnier les cygnes. [...] je suis consacré au même dieu, $[. .$.$] je tiens de notre maître un$ don prophétique qui ne le cède pas au leur (1965, XXXV, 84, p. 140).

Dans l'Antiquité et pour l'Église, les oiseaux sont des êtres intermédiaires entre l'humain et ce que Platon appelle les « choses divines », vers lesquelles existent trois voies d'accès :

- l'interprétation des mythes, qui nous présentent quelque chose de l'origine, cet âge d'or durant lequel les dieux marchaient parmi les hommes;

- les quatre fureurs de la priska theologia par lesquelles l'esprit divin descend en nous et nous met dans l'état d'enthousiasme ou possession divine: fureur poétique (inspirée par les Muses), mystériale (l'ivresse, Dionysos), prophétique (Apollon) et érotique ;

- le discours «surhumain » circulant dans une assemblée composée des meilleurs : le tout valant mieux que l'ensemble des parties, ou le quatuor que les musiciens individuels, c'est ce qu'essaient de reproduire les académies.

Le cygne était l'emblème de la fureur prophétique; chanter Il bianco e dolce cigno réunit ces trois modes, réinterprétés par des hommes et des femmes profondément chrétiens, pour lesquels les fureurs hérö̈ques résonnent avec l'excessus des Sermons de saint Bernard (1992), désir de sortie de l'humaine condition par la pratique de l'excès et sortie de soi. Ce qui meurt et renaît est à leurs yeux un quotidien réellement présent (ne serait-ce qu'à la messe) et a davantage encore que dans l'Antiquité valeur de mystère révélé.

Il bianco e dolce cigno est construit sur la comparaison entre un cygne mourant, un poète mourant et leurs sensations. Cette compréhension entre "mort» et "petite mort», à la jointure de la joie, du désir et de la mort, se retrouve dans la plupart des madrigaux des académies.

Il bianco e dolce cigno cantando more Ed'io piangendo giung'al fin del viver mio

\section{Stran'e diversa sorte \\ Ch'ei more sconsolato \\ Ed'io moro beato \\ Morte che nel morire \\ M'empie di gioia tutto e di desire}

Se nel morir, altro dolor non sento

Di mille volt'il dì sarei contento.

Le doux cygne blanc chantant meurt Et moi pleurant j'atteins la fin

[terme + but] de ma vie.

Sort étrange et différent

Que lui meure inconsolé

Et moi je meurs content

Mort qui dans le mourir

M'emplit entier de joie et de désir

Si en mourant je ne sens autre douleur Je serais content de mourir mille fois par jour!

Nous avons divisé le poème en trois sections ${ }^{11}$. Le premier distique reprend le thème mis en vogue par Martial (cité dans Morisset et Thévenot, 1978, p. 1008) : 
Dulcia defecta modulatur carmina lingua

Cantator cygnus funeris ipse sui

À langue défaillante il module de doux chants

\section{Le cygne chanteur de}

ses propres funérailles

Contrairement au cygne de Martial, l'oiseau meurt en deux mots (cantando more). Les deux syllabes de more sont traitées de manière calme et conclusive, sur une cadence parfaite. Le poète pleure de mourir, et cette fin dure: les deux premiers vers sont verb-oriented, l'accent est sur le viver', c'est l'action qui importe, non la fin.

La mort passe au ralenti, en cinq mots, sur un quadruple contretemps, dans une attente prolongée, puis une cadence abrégée suivie d'un silence. L'effet est accentué par l'étendue inhabituelle du vers, des valeurs longues et une surprenante modulation en fa suivie d'une descente dans le grave. L'impression est celle d'un temps prolongé. Giung' allonge encore ce temps en lui supposant une attente préalable et la suggestion d'un plaisir; al fin signifie «enfin!», dans un soupir. Le fin est à double sens: cette fin est aussi un but. Un io quadruple commence et finit le vers grâce au jeu sur mio, et la phrase passe de l'un à l'autre via l'expression d'une douleur et un changement de mode, quand, à la reprise du piangendo, le fa du soprano monte à «la». Cette modulation ouvre sur une montée acoustique et une autre harmonie, en répétant une phrase identique: io (je), passé par douleur dans une mort lente, peut-être désirée, n'est plus le même quand il revient.

La seconde section inverse la première opposition. Le cygne (après tout, ce n'est qu'une bête) meurt inconsolé (de perdre la vie, son seul bien), alors que le poète meurt content puisque sa mort est un passage, et non une fin : la lente entropie s'est muée en renouveau. Nous sommes passés sous le signe de la quatrième fureur, l'Eros. Tout ce que ressent ce $\backslash j \mathrm{j} \backslash$ quadruple est sensuel. Le moro beato s'élève à un hiatus béant, l'allitération en $\backslash \mathrm{m} \backslash$ (morte che nel morire/m'empie) est une attente de jouissance mortelle (gioia, le jö̈ des troubadours, exaltation vitale et mortelle, sortie de soi), à laquelle se mêle la souffrance des voix masculines tendues par la longueur et la hauteur inconfortables de leur phrase musicale. En passant dans la relative mineure, c'est-à-dire en ton de mi, mode de ré, avec sensible, puis en retombant en ton de sol, même mode mais en conservant la sensible, la seconde partie du poème monte à un sommet de malaise et de plaisir : montée inconfortable et sommet périlleux, douloureux pour les voix d'hommes, qui s'effectue sur les mots $e d$ io moro beato. Puis c'est la redescente, avec le changement qualitatif opéré par l'extase, car l'on ne redescend pas identique d'une telle montée. Cette seconde section imite en diminutions le leitmotiv de la première, sol sol sol la la si, cette fois sans virgule, en noires. L'allongement final de morire souligne à nouveau l'action, et non sa fin. Ce mourir a pour effet d'emplir le poète de « joie» et « désir » sur une phrase musicale descendante et conclusive, un retrait après suggestion de béatitude.

Cette section commente la supériorité du poète : la mort de l'animal est désespérée parce que pressentie sans retour; elle met en valeur celle du poète. Deux séquences de deux vers encadrent un sommet sur ed io moro beato. Le premier vers, scandé à l'antique par un dactyle suivi d'un ana-

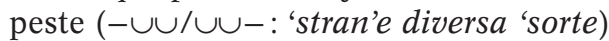
accentue les mots stran' et sorte en une harmonieuse récurrence des sons $\backslash s \backslash, \backslash r \backslash$, $\backslash t \backslash$ sur des voyelles claires. Les accents du texte poétique sont contrariés par les accents musicaux et les variations en hauteur des sons: cela monte sur les temps faibles, cela descend sur les temps forts. Le deuxième vers est heurté et sombre, avec un more sconsolato en $\backslash \hat{\backslash} \backslash$. Le vers central répète le verbe mourir, non plus au subjonctif mais à l'indicatif, passant du potentiel à la réalité en train de se vivre mourir.

À ce passage correspond, à notre oreille, l'introduction d'une sensible, le passage d'une écriture modale à une écriture tonale, la certitude d'une fin attendue retardée, un jeu prolongeant le jeu. De ce point de vue anachronique, dans ce madrigal, la modalité sculpte un instant continu, alors que le recours au langage «tonal» amène l'exacerbation d'une conscience qui se sent mourir. En deux endroits, trait archaïque, le ténor fonctionne comme une teneur, que les autres voix commentent: il véhicule la souffrance et l'extase de la "petite mort», et la joie dans les deux mesures finales. L'impression d'incertitude que ressent aujourd'hui un auditeur devant cette pièce ni modale ni tonale contredit un préjugé selon lequel la tonalité aurait un jour triomphé de la modalité comme l'avion à réaction sur l'avion à hélices. D'autre part, ici, deux procédés expressifs expriment l'un, la permanence et l'éternel retour, l'autre la durée linéaire, l'attente de la fin. Le système n'est pas structuré par la musique, mais par le poème.

La dernière section, exclamative et joyeuse, est une conclusion qui réexpose l'explication précédente en reprenant sur altro dolor non sento la formule $-\cup \cup / \cup \cup-$.
Comme un alleluia, elle associe la joie à la vie régénérée. Comme le cygne, le poète est seul. L'absence d'autre est une caractéristique de ce poème où le locuteur anonyme est comparé à un être situé à un autre degré de l'échelle du vivant et s'adresse à d'autres lui-même. Seul moment homophonique de cette œuvre, cette section prépare par l'immobilité le feu d'artifice du dernier vers, sur une musique joyeuse en valeurs brèves, avec un rythme pointé rapide, et des paroles exprimant la joie de mourir, mais au conditionnel, non au futur: Di mille morte il di sarei contento (je serais content de mourir mille fois par jour). Ce madrigal est une sorte d'emblème érotique relié à l'essentiel, à ce qui meurt et qui renaît, et au-delà de l'allusion au mystère de la résurrection, à un renouveau du cosmos et de la société.

\section{" MOURIR » DANS LE MADRIGAL: LE BAISER DE LA CHOSE}

La question de la Chose ne hante pas seulement la psychanalyse, elle sous-tend toute démarche de recherche, tout désir de remonter à la source authentique, elle est celle de l'origine comme condition de possibilité inatteignable de tout ce qui nous est constitué comme «monde». Elle est présente dans le concept platonicien de pragma, dans le nouménal kantien, dans la «chose» heideggérienne, avec cette particularité d'être l'espoir de la vérité et de la plénitude qui s'avère devoir être acceptée comme absolument inatteignable, parce qu'elle n'est qu'un manque, un rien ${ }^{12}$.

C'est à partir de sa lecture croisée de Freud (Au-delà du principe de plaisir, 1951 et l'Entwurf, 1956) et de Kant (Critique de la raison pratique, 1980) que Lacan, dans le Séminaire, Livre VII (1986), dégage la notion de Chose et défriche sa conception de la jouissance: quel est donc ce champ ouvert par Freud sous le nom de das Ding (la chose), qui se retrouve à la fin de sa recherche dans $A u$-delà du principe de plaisir, quand il tente de désigner «ce qui dans la vie peut préférer la mort ${ }^{13} »$. Le champ de la jouissance et de la Chose se pose d'abord sous le signe d'un rapport au mal et à la mort.

La théorisation lacanienne tente de dégager la structure de ce questionnement moral, en donnant à la Chose une place dans le processus du désir du sujet de l'inconscient, comme effet de langage: la Chose, ce qui pâtit du signifiant (Lacan, 1986), est ce dont le langage, par sa nature symbolique, nous coupe, d'un geste de «castration symbolique». La condition humaine est marquée par sa prise dans le signifiant, et du coup soumise à sa loi: le symbole est la représentation d'une absence; il n'est pas la Chose, il la «tue» 
Jacques Ancadelt : Il liance e dalce cigno (verise gardano 154)) (original 1539) suig cicinale

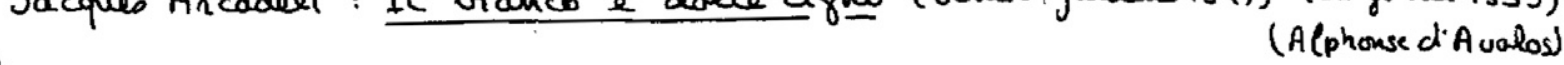

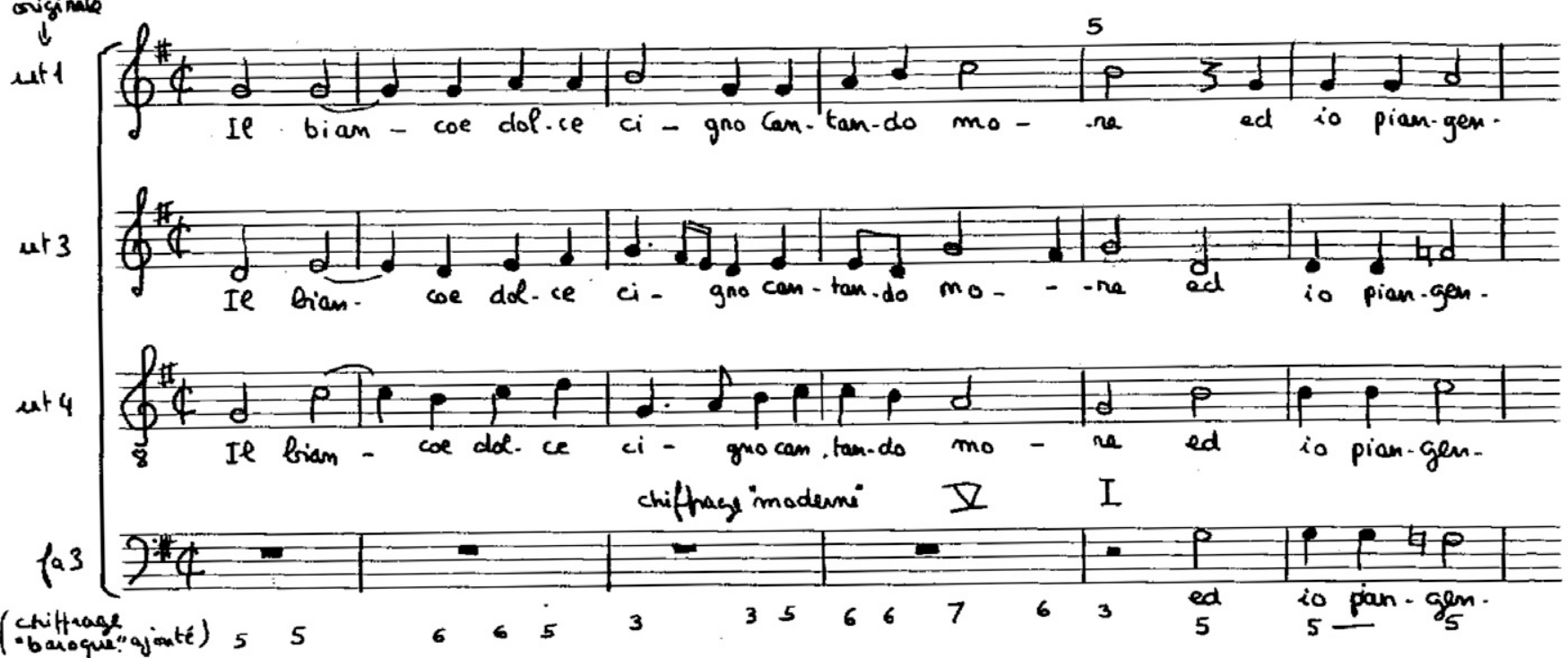

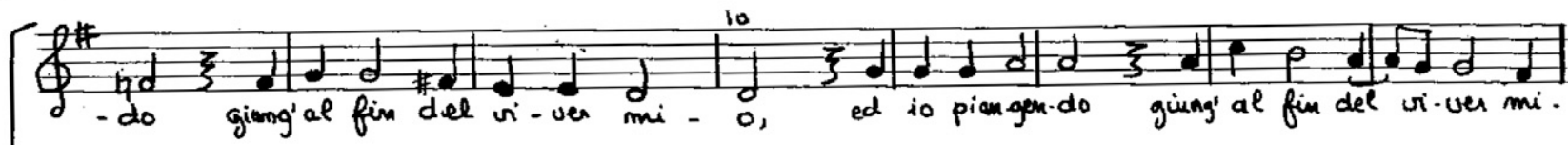

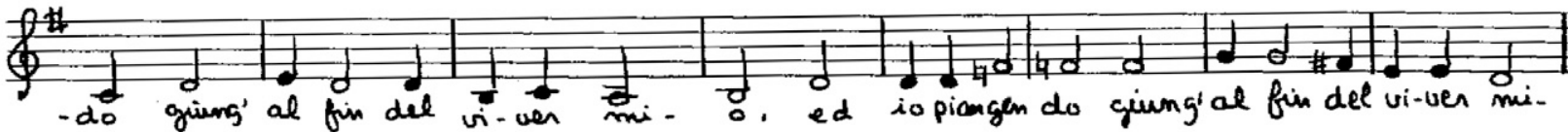

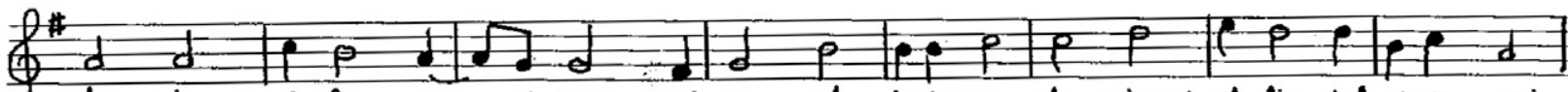
\&-do giung' al fire del vi-ver sui.o, ed iopiengen-do giung' al fir del viver mi-

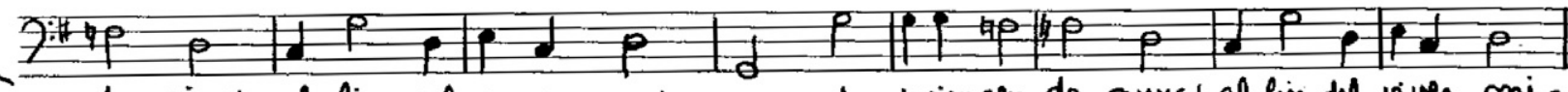

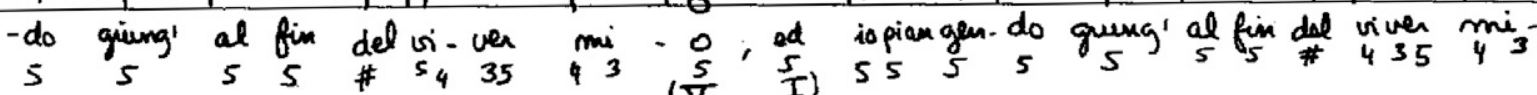

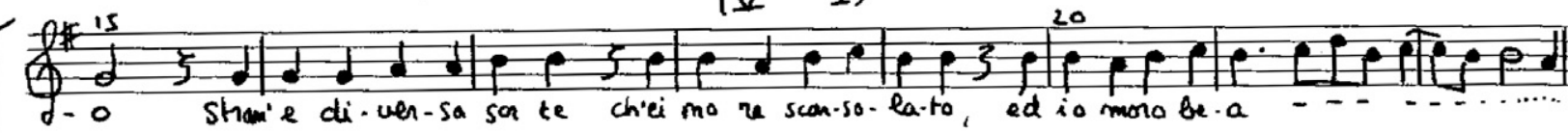

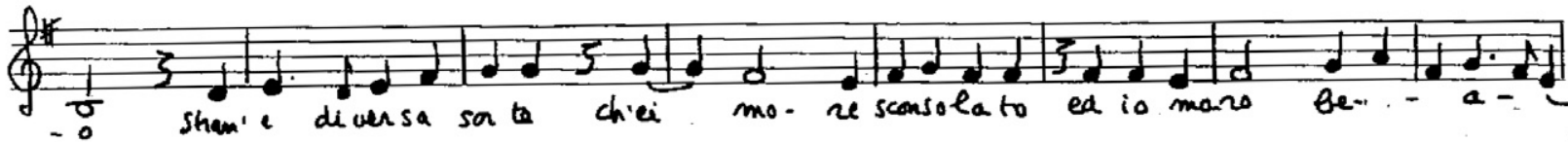

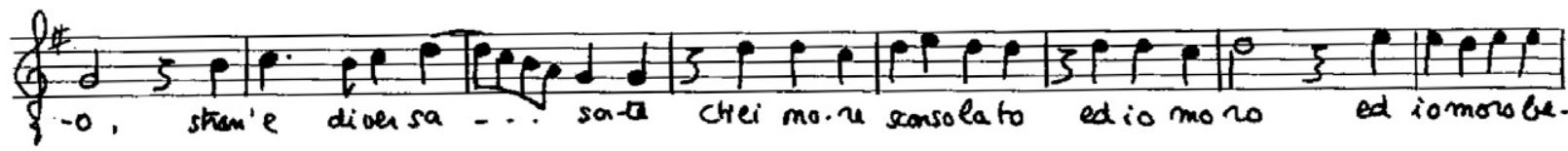

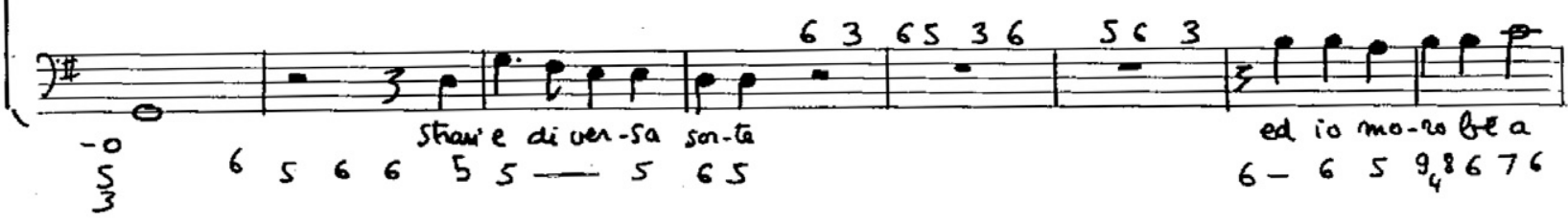




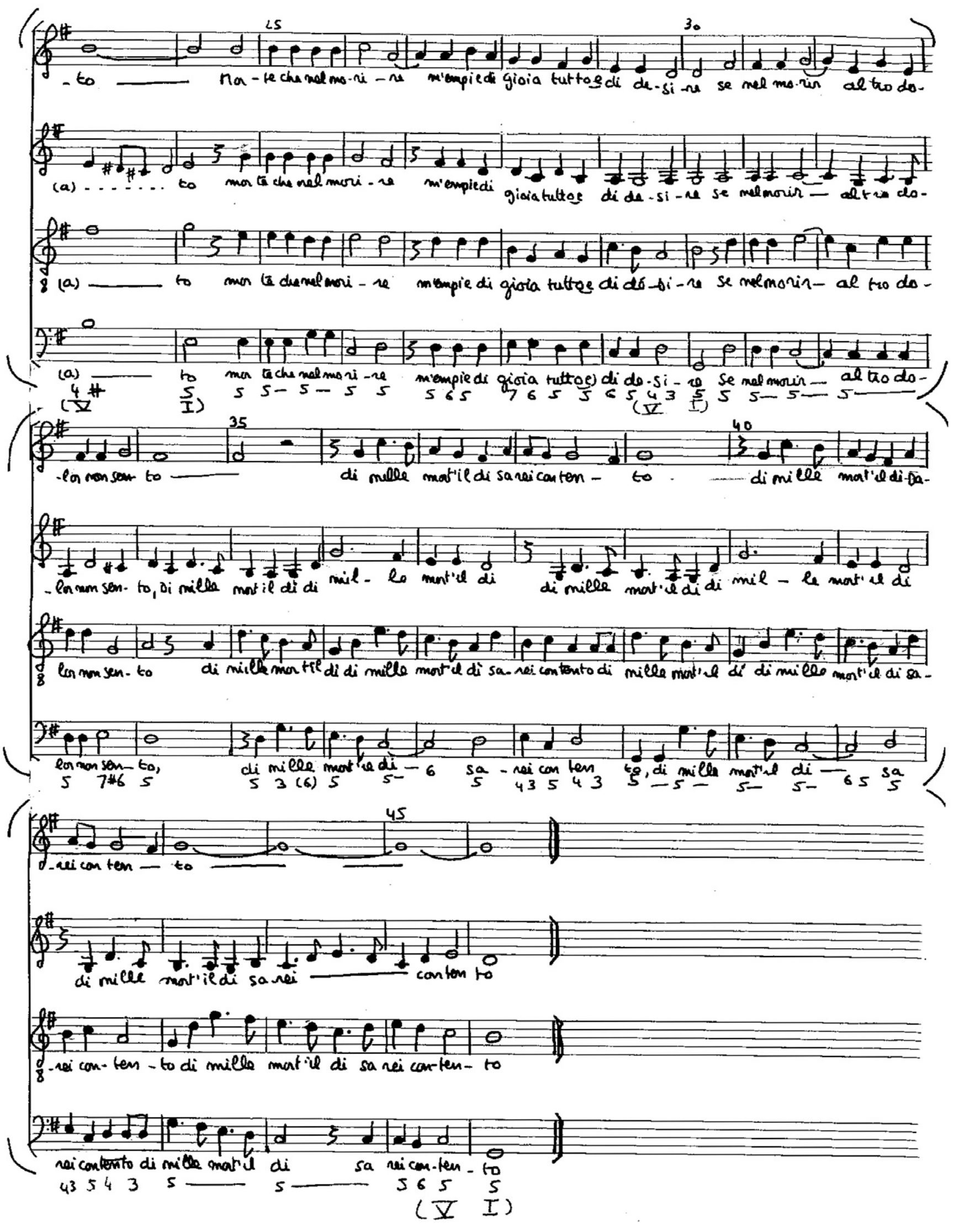


en la disant mais dans le même temps, il nous permet l'accès signifiant au monde, condition de possibilité d'un sujet humain désormais marqué par la perte de ce que le signifiant ne peut que re-présenter, voire seulement évoquer ${ }^{14}$.

Ainsi, la Chose reste comme perte, rien qu'une perte et perte de rien, manque nécessaire, lieu vide, impossible, indicible, irreprésentable, que l'être humain s'ingénie à emplir de ses mythes et constructions imaginaires, à figurer, à habiller, à connaître, à conjurer, à nier, à incarner. Ce vide promis comme plein serait la source de toutes les créations humaines. Comme la mort, elle pose la question de l'irreprésentable, de l'inconnaissable, de l'inexpérimentable.

La jouissance est directement liée à la Chose, comme le désir; elle est tournée vers elle, sauf que le désir s'articule à la loi du signifiant et supporte le manque de la Chose. La jouissance est ce qui conteste la loi, la nie, la refoule, la transgresse dans l'illusion de la Chose, promesse de plénitude et d'anéantissement. C'est une autre satisfaction, qui relève d'une détermination inconsciente sans rapport ni avec le plaisir conscient et "vivable», ni avec le désir articulé à la loi, et qui apparaît souvent comme une contrainte insensée et morbide à l'œuvre dans les symptômes et le délire. Elle pose la question des limites, du franchissement et de l'impossible, et se met en contradiction avec un principe de vie et de conservation de soi. C'est un concept omniprésent en psychanalyse, qui différencie plusieurs formes de jouissance, dont la jouissance sexuelle, laquelle renvoie, mais du côté du possible, à cette notion de franchissement des limites du dicible et de l'indicible (voir Braunstein 2005).

\section{LE MADRIGAL:}

\section{MORT ET «PETITE MORT»}

À partir de là, voyons la singularité du «mourir» dans le madrigal que nous étudions. Il s'agit à la fois de la possibilité de franchir le seuil de la mort et de revenir, et cela sous la forme d'une extase languissante. En effet, l'analyse du madrigal le montre de différentes manières et la forme de l'interprétation vocale l'accentue, la mort est ici une extase des sens dont on se relève transformé.

Mourir m'emplit tout entier de joie et de désir: le contexte est celui d'une transgression de la loi naturelle, dans ce jouir de mourir. Dans ce madrigal apparaît la joie du départ et du retour depuis la mort, avec cette sorte de volupté du va-et-vient d'un état à l'autre, du passage par un anéantissement rémissible du sujet qui évoque, à plus d'un titre, la jouissance sexuelle.
L'anéantissement du sujet comme signifiant, qui caractérise la jouissance dans les symptômes cliniques, ne prend pas ici une dimension d'horreur face au néant. Tout juste y est décrite l'envie d'y revenir, mais davantage pour insister sur l'extase que pour évoquer la souffrance ou la hantise de ne pouvoir s'en passer. L'anéantissement du sujet est pensé comme une pâmoison. Il semble que ce «mourir» vacille entre plaisir et jouissance: il n'y a pas réellement de souffrance ou de contrainte. Nous ne sommes pas non plus dans le registre de l'apaisement des tensions qui signe le plaisir: les voix masculines, cela a été souligné, sont poussées à leur limite, et avec elles une forme de douleur s'étire, au rythme de cette lente entropie; puis la présence des larmes et de la joie accentue l'intensité contradictoire et confuse de la scène.

Il y a là, convoquée, toute la puissance tumultueuse d'Eros, non altérée par la négativité radicale et séparatrice de la mort. La jouissance du madrigal pourrait être tout entière du côté de la jouissance sexuelle, juste là, entre la jouissance inconsciente du symptôme et le plaisir.

\section{LE MADRIGAL ET LA CHOSE:}

LE SECRET ET LE SILENCE

Ce sont deux motifs du dispositif du madrigal: pour chanter, les madrigalistes se mettent en cercle et sont couverts, liés, «connectés » l'un à l'autre par un simple toucher sur l'épaule: au milieu du cercle, tenu secret... le vide. Le groupe de madrigalistes se rassemble «tout contre» le bruit du monde, c'est-à-dire qu'il détache du bruit du monde une parcelle d'un silence qui doit être parfait. Dans le vide ainsi recueilli par le cercle qui le crée $e x$ nihilo, de cet étrange corps polyphonique, naît une voix faite de voix, qui se fait et se défait, et révèle par cet enchevêtrement l'architecture de l'harmonie du monde. Ce n'est pas la Chose au centre qui parle car elle n'est qu'un vide, mais ce vide vibre des voix qui tentent de «la» révéler et de l'atteindre. Ainsi la Chose lacanienne, révélée par un signifiant qui la rate toujours d'essayer de la dire.

Mais là encore, le poids morbide de la négativité fait manque: le madrigal joue des oppositions, mais elles co-existent; le silence, à l'intérieur du morceau lui-même, n'est pas un moindre bruit, il est en positif, il fait partie de la musique. La mort est possible et surmontable, le vide et le silence permettent de communiquer avec la force harmonieuse de la nature divine.

\section{LE MADRIGAL:}

LA DIFFÉRENCE CONTRE LA NÉGATIVITÉ

Jusqu'ici, et c'est là que le madrigal ne se laisse pas tout à fait saisir, il semble à la fois jouer avec le manque, le trou central, et faire valoir par les contrastes et le contrepoint toutes les oppositions, non pas en négatif, mais en positif, à la manière d'une mosaïque où chaque élément ne se détache pas du fond, mais de sa différence avec un autre élément. Pensez au symbolique chez Lacan: le signifiant n'existe que pour se différencier d'un autre signifiant, différence dont l'un des paradigmes, celui de la différence entre l'homme et la femme, traverse de part en part les cultures humaines. Sur le corps commun, la détermination sexuelle s'inscrit comme une coupure ou séparation nécessaire à la possibilité de la pensée et du sens.

Là encore le madrigal, qui parle d'amour avec légèreté, réunit des hommes et des femmes «à égalité », dans un même corps, et fait montre de sa singularité: le paradigme de la différence se noue dans un corps, les hommes et les femmes ne dialoguent pas, n'incarnent pas un homme ou une femme sous forme d'un personnage, ils se lient et délient l'un de l'autre et décuplent ainsi la palette sonore de la voix. Dans cette volonté de faire totalité, où même la coupure symbolique fondamentale entre les sexes ne divise pas, il y a tout de même une trouée, une percée centrale, où le rapport à l'Autre comme transcendance divine prend sa source. Le trou n'est pas synonyme de mort, mais de présence divine, la mort n'étant qu'un moyen d'y goûter.

Tout cela semble vouloir évoquer une relation privilégiée des madrigalistes à l'Au-delà par la pratique rituelle.

Voilà un cas singulier pour nous: là où notre époque semble se confronter à la question du néant, de l'absurde, de la finitude; là où notre "science ", en se focalisant sur le réel de la mort, tend à taire ou à réduire son impact imaginaire et symbolique; là où, en suivant le fil de la mort du cygne et de la sagesse socratique, on aurait pu s'attendre à découvrir la mort sereine et assumée du sage, voici que le madrigal en fait une expérience initiatique érotique, et représente la situation toute particulière de cette aristocratie déclinante recherchant la quintessence d'elle-même.

À l'instar de ce qui a été thématisé ici sur l'évolution du madrigal dans son contexte politique, il faudrait continuer l'analyse du thème de la mort dans la musique baroque: P. H. Frangne (2002), situe le changement de la pensée sur la mort ${ }^{15}$ au moment de la philosophie de Hegel, le penseur inaugural de la négativité ${ }^{16}$, changement déjà sensible pour lui dans les modifications introduites au tournant du XVII ${ }^{\mathrm{e}}$ siècle par Monteverdi dans ses polyphonies, notamment dans le Combattimento. Pour cet auteur, l'amour et la mort n'y sont plus 
racontés de la même manière par le chant et le récit: alors que face à la mort, dans le madrigal d'Arcadelt le héros soupire, chez Monteverdi, il tremble doublement, puisque Monteverdi introduit des tremblements dans l'interprétation vocale ellemême.

C'est aussi en se référant au Combattimento que Guiomar, dans ses Principes d'une esthétique de la mort (Guiomar, 1967), remarque un tournant dans le traitement du thème de la mort; cette thématique prend d'après lui une place de plus en plus imposante: Guiomar part d'une différenciation entre un art de divertissement au sens pascalien, qui écarte la possibilité d'une esthétique de la mort et cherche à la conjurer ou à s'en défendre, et un art qui met la Mort en jeu. Il décrit comment, petit à petit, dans les textes des madrigaux italiens, la mort prend le pas sur le divertissement, le tragique sur le dramatique, depuis le poème d'amour léger, voire paillard, des premiers madrigaux, jusqu'à la mise en scène de la lutte entre la Vie et la Mort dans Monteverdi. L'émergence dans un même mouvement de l'opéra, de l'absolutisme et du traitement baroque de la mort poursuit un travail de sape, jusqu'à engloutir les ruines de la polyphonie à voix égales et de l'aristocratie.

\section{Bibliographie}

ALBERTI, L.B. (1639). Discorso dell'origine delle Accademie publiche e private e sopra l'impresa degli affidati di Pavia, Gênes.

ANONYME ITALIEN (16 $\mathrm{e}$ S.). Le concert champêtre, Musée du Berry, Bourges.

ARTUSI, G.M. (1596). L'Artusi overo delle imperfettioni della moderna musica, Florence.

BANDELLO, M. (1990 [1540-1573]). Novelle, Milan, Rizzoli.

BRAUNSTEIN NESTOR, A. (2005). La jouissance - Un concept lacanien, Ramonville-Saint-Agne, Erès, coll. «Point - Hors ligne».

CASTIGLIONE, B. (2002 [1528]). Il Cortigiano, Venise, 1528; Milan, Mondadori.

CERONE, P. (1969 [1613]). Il melopeo y maestro, Naples, 1613; Bologne, Arnaldo Forni.

CHAILLEY, J. (1950). Histoire musicale du Moyen Âge, Paris, PUF.

CINTHIO, G.G. (1999 [1565-1574]). Hecatommiti, Venise, 1565-74; Milan, Rizzoli.

DE CLAIRVAUX, B. (1992). Euvres mystiques, trad. du Traité de l'amour de Dieu et des Sermons sur le Cantique des cantiques, trad. de A. BÉGUIN, Paris, Seuil.

DELLA CASA, G. (1988 [1558]). Il Galateo, Firenze 1558; Milan, BUR.
DONATI, C. (1988). L'idea di nobiltà in Italia (secoli 16-18), Bari, Laterza.

DONI, A.F. (1965 [1544]). Dialogo della musica, Vienne, Malipiero.

FRANGNE, P.H. (2002). "L'art et la mort, Hegel et Monteverdi », conférence, Paris.

FREUD, S. (1956). «Entwurf einer Psychologie ", dans La Naissance de la psychanalyse, lettres à Wilhelm Fliess, notes et plans, Paris, PUF, p. 307-396.

FREUD, S. (1951). "Au delà du principe de plaisir ", dans Essais de psychanalyse, Paris, Payot, p. 76-162.

GLAREANUS, H. (1967 [1547]). Dodecachordon, Bâle, 1547; New York, Broude Bro.

GRAZZINI, A.F. (dit IL LASCA) (1989 [1566]). Le Cene, Milan, Rizzoli.

GUAZZO, S. (1574). La civil conversatione, Brescia, V. Sabbio - T. Bozzola.

GUIOMAR, M. (1967). Principes d'une esthétique de la mort, les modes de présences, les présences immédiates, le seuil de l'Au-delà, Librairie José Corti.

KANT, E. (1980 [1788]). Critique de la raison pratique, Paris, Gallimard.

LACAN, J. (1986). Le Séminaire, livre VII, L'éthique de la psychanalyse, 1959-1961, texte établi par J.-A. MILLER, Paris, Seuil.

MORISSET, R. et G. THÉVENOT (1978). Les Lettres Latines, Paris, Magnard.

NEWCOMB, A. (1980) The Madrigal at Ferrara 1579-1597, Princeton, New Jersey, Princeton University Press.

PLATON (1965). Phédon, traduction de Chambry, Garnier-Flammarion.

TÉNOUDJI, P. (2002). Harmonie perdue: le madrigal polyphonique italien du rituel à la représentation $\left(X V I^{e}-X V I I^{e}\right.$ siècles), ou comment penser ensemble individu, musique, société, monde, Presses universitaires de Strasbourg.

TOCQUEVILLE, A. DE (1958 [1833]). Voyages en Angleterre, Iralnde, Suisse et Algérie, texte établi et annoté par J.-P. MAYER et A. JARDIN, Paris, Gallimard.

VECCHI, O. (1984 [1596]). L'Amfiparnaso, Bologne.

VINCENTINO, N. (1959 [1555]). L'antica musica ridotta alla moderna prattica, 4 vol., Rome, A. Barre, 1555 ; Kassel, Bärenreiter.

\section{Notes}

1. Analyse détaillée et reproduction de ces images dans Ténoudji, 2002.

2. "Le madrigaliste ne chante pas à pleine voix, mais artistement, en voix de tête ou fausset. » (Cerone, 1613, p. 13.)

3. Voir Le concert champêtre, anonyme italien $\mathrm{du} \mathrm{XVI}^{\mathrm{e}}$ siècle.

4. Voir Bandello, 1540; Grazzini, 1566; Cinthio, 1565.
5. Voir Castiglione, 1528; Della Casa, 1558; Guazzo, 1574

6. Voir Donati, 1988.

7. Voir Doni, 1544; Glareanus, 1547 ; Vincentino, 1555.

8. Cette thèse a notamment été défendue par Alberti (1639, vol. 2, p. 110); elle est reprise par Newcomb, 1980, et Donati, 1988 (voir aussi Ténoudji, 2002).

9. Notamment Gorgias (523e, seq.); voir également La République (L.VI, sur les «gardiens »).

10. Je pense à la professionnalisation des musiciens des cours, à l'Académie française ou aux académies italiennes actuelles.

11. Pour rester fermes dans notre propos, l'analyse alterne, incomplètes, explication de texte et analyse musicale.

12. Pour une étude détaillée de la Chose, chez Freud et Lacan, mais aussi dans la philosophie et les mythes, lire le cours de Pavon Cuellar, Université de Paris VIII (2003-2004).

13. «C'est au niveau de la bonne et de la mauvaise volonté, voire de la préférence pour la mauvaise au niveau de la réaction thérapeutique négative, que Freud, au terme de sa pensée, retrouve le champ de das Ding, et nous désigne le plan de l'au-delà du principe de plaisir. C'est comme un paradoxe éthique que le plan de das Ding est retrouvé à la fin, et que Freud nous y désigne ce qui dans la vie peut préférer la mort. Et il s'approche par là plus qu'aucun autre, du problème du mal, plus précisément, du projet du mal comme tel. » (Lacan, 1986, p. 124.)

14. Il ne peut que l'évoquer car le langage est un système de références qui a sa logique en luimême: en prononçant un mot, on ne rend pas présente la chose qu'il désigne; la linguistique saussurienne, dont Lacan s'inspire, différencie à l'intérieur du mot le signifié comme idée de la chose et le signifiant, comme image acoustique: le mot re-présente la chose, il vient en "place de », mais il n'est qu'un mot, c'est-à-dire une variable sonore qui par son étoffe évoque toujours en même temps une chose et d'autres choses (ex: araignée - a régné).

15. Dans le sens de dégager, comme nous avons essayé de le faire, la négativité comme point pivot.

16. «Cette philosophie peut être considérée comme la première philosophie authentique de la mort, comme une véritable philosophie de l'inquiétude » (Frangne, 2002, introduction de la conférence). 